\title{
Biochemical urinalysis of healthy kidney and stone- generating kidney in unilateral urolithiasis
}

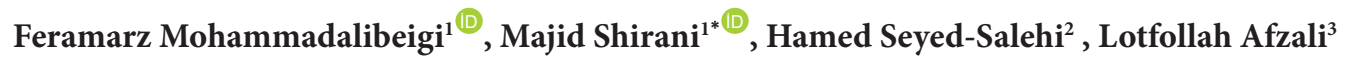 \\ ${ }^{1}$ Department of Urology, Shahrekord University of Medical Sciences, Shahrekord, Iran \\ ${ }^{2}$ Student Research Committee, Shahrekord University of Medical Sciences, Shahrekord, Iran \\ ${ }^{3}$ Department of Surgery, Shahrekord University of Medical Sciences, Shahrekord, Iran
}

\section{A R T I C L E I N F O}

Article Type:

Original

Article History:

Received: 12 October 2018

Accepted: 19 December 2018

Published online: 20 January 2019

Keywords:

Unilateral urolithiasis

Biochemical urinalysis

Kidney stone

Chronic kidney disease

\begin{abstract}
A B S T R A C T
Introduction: Oxalate, calcium, uric acid, and citrate are among the most widely known biochemical factors for urinary stone formation. In most cases, urolithiasis occurs as unilateral despite the role of systemic metabolic factors in kidney stone formation.

Objectives: The present research aimed to compare these urinary biochemical factors in healthy and stone-generating kidneys in patients with unilateral urolithiasis.

Patients and Methods: Forty patients with unilateral urolithiasis participated in this cross-sectional, descriptive-analytical study. The patients were hospitalized in kidney surgery and kidney urology ward of Kashani hospital of Shahrekord, southwest Iran. After the implementation of crushing stone using transurethral lithotripsy (TUL), 5-10 cc of urinary sample was collected from each kidney. An AutoAnalyzer (Mindray Company, Bs-360 model) and laboratory kits (Bionic Company) were used to measure calcium, uric acid, and creatinine. Moreover, a manual method and Darman Faraz Kave company kits were applied to measure the levels of oxalate and citrate. Results on the healthy and stone-generating kidneys of each patient were separately analyzed using the Stata 13 software.

Results: The difference in the mean ratio of uric acid, oxalate, and citrate to creatinine in the healthy kidneys and stone-generating kidneys was not significant. However, the mean UCa/UCr ratio in the healthy kidneys was $0.27 \pm 0.07$ and relatively greater than that in the stone-generating kidneys $(0.11 \pm 0.04) \quad(P=0.06)$.

Conclusion: The studied topical factors and secretory disorders had not any significant relationship with unilateral urolithiasis. The cause of unilateral urolithiasis should be searched in other factors such as metabolic factors, main positions of individual during 24 hours and sleeping, and anatomic disorders of kidney stone, or multifactorial.
\end{abstract}

\section{Implication for health policy/practice/research/medical education:}

The cause of unilateral urolithiasis should be searched in other factors such as metabolic factors, main positions of individual during 24 hours and sleeping, and anatomic disorders of kidney stone, or multifactorial.

Please cite this paper as: Mohammadalibeigi F, Shirani M, Seyed-Salehi H, Afzali L. Biochemical urinalysis of healthy kidney and stone-generating kidney in unilateral urolithiasis. J Renal Inj Prev. 2019;8(2):151-156. doi: 10.15171/jrip.2019.28.

\section{Introduction}

There is an increasing prevalence of urinary stone around the world (1). The reported prevalence rates vary between 68 and 720 cases per 1000000 population depending on the country or the region. Besides that, it can be affected by genetic, nutrition, and geographic and socioeconomic factors (2,3). Urinary stones occur in 8\%-15\% of Europeans and North Americans in their lifetime (4).

The prevalence of urinary stone is estimated at $5.7 \%$ while the western toward southwestern regions of Iran have the highest number of patients with kidney stone (5).

Nephrolithiasis is a systemic disorder that is associated with chronic kidney disease, bone loss and fractures, increased risk of coronary artery disease, hypertension, type 2 diabetes mellitus, and metabolic syndrome (6). Early diagnosis and treatment of kidney and urinary tract stones and their excretion can prevent the enlargement of stone and associated complications (7).

The occurrence of urolithiasis is three times higher in men than in women (8). The pathogenic mechanisms of kidney stone formation are complicated and entail metabolic and environmental risk factors (6).

The most effective biochemical factors in urolithiasis include citrate, oxalate, calcium, phosphate, sodium, 
potassium, uric acid, magnesium, urinary $\mathrm{pH}$, and urea, all of which are compared with urinary creatinine ratio. Among them, oxalate, calcium, and uric acid are comparatively more important. Supersaturation of these factors causes a significant increase in the risk of kidney stone formation and its recurrence $(9,10)$.

The metabolic predisposition of kidney stones is mainly due to increased excretion of crystals such as calcium, uric acid, oxalate, and cysteine in urine or the reduction of kidney stone formation inhibitors such as like magnesium, citrate or pyrophosphate (11).

Traditionally, a urinary calcium/creatinine ratio (UCa/ $\mathrm{UCr}$ ) of $>0.21$ is considered abnormal and indicative of hypercalciuria. However, recent studies have shown that $\mathrm{UCa} / \mathrm{UCr}$ varies depending on age and geographic area (12). The most common type of kidney stones contains calcium worldwide $(8,13)$.

Eighty percent of urinary calculi are calcium compounds, $62 \%$ of which are mainly seen as calcium oxalate (14), and the remainder consists of uric acid (5-10\%), struvite or carbonate apatite (secondary to infection), cystine $(1 \%)$ and rare stones $(4,14)$. Acidic urine, hypercalciuria, and hyperoxaluria are risk factors for calcium oxalate urolithiasis $(15,16)$. Unilateral hypercalciuria should be considered a risk factor for nephrolithiasis in children with recurrent stone episodes in one kidney only (17).

In a study, a significant increase of oxalate/creatinine ratio and $\mathrm{UCa} / \mathrm{UCr}$ ratio among patients with urinary stones in comparison with normal population was seen. Moreover, the citrate/creatinine ratio and magnesium/ creatinine ratio in those with kidney stone decreased significantly in comparison with those without kidney stone. There was no significant difference in phosphate/ creatinine ratio between people with kidney stone and those without (18).

Recurrent stone formers commonly present with calculi on the same side, while the etiology of recurrent unilateral urolithiasis is unknown yet (19). The physiochemical mechanism of nephrolithiasis has not been well understood at the molecular level, while this issue is important in terms of the control and prevention of renal stone formation (20).

Despite different explanations for urolithiasis, it has not yet been clarified why urolithiasis mainly occurs as unilateral, and why large stones are formed in some patients and small recurrent stones are formed in some others.

The body system is affected by metabolic factors through blood flow. Both kidneys have the same blood flow regarding volume and compounds. Therefore, metabolic disorders may influence the whole urinary system similarly.

It is expected that kidney stone occurs in both kidneys, however, the statistics demonstrate that for the patients with known metabolic causes of kidney stone formation, urolithiasis occurs mainly as unilateral. Several theories and reasons for these inconsistencies have been presented (19-21).

\section{Objectives}

The present study was conducted to compare the biochemical factors of the healthy kidneys and stonegenerating kidneys in patients with unilateral urolithiasis.

\section{Patients and Methods \\ Patients}

This cross-sectional, descriptive-analytical study was carried out in the spring and summer of 2015. Forty patients participated in this study. The patients suffered from unilateral urolithiasis and were hospitalized in the urology and kidney surgery ward of Kashani hospital of Shahrekord, southwest Iran. The patients were candidate for crushing stone with transurethral lithotripsy (TUL). The sample size was derived 30 by a sample size calculation formula, $95 \%$ confidence interval, and $15 \%$ precision. To enhance the rigor of the study, 40 patients were enrolled.

The inclusion criteria were definite diagnosis of unilateral urolithiasis with a healthy kidney based on the results of sonography conducted by a radiologist. The exclusion criteria were the bilateral urolithiasis, severe metabolic disorders, high blood pressure, the history of metabolic bone diseases, the history of steroids or diuretic drugs consumption, and the lack of or insufficient physical activity.

The patients' demographic characteristics (age, gender, and living place) and history were recorded at admission. Either spinal anesthesia methods or general anesthesia was used after the patients were transferred to the operating room, based on their conditions and the anesthesiologist comment. To excrete the urinary calculi, the TUL method was used. In this method, urethra scope was penetrated into the bladder by the urinary tract and, after going up through the ureter and reaching stone, crushed the stone by one of the intra-organ crushers consisting of pneumatic lithotripsy or lithoclast, laser, ultrasonography or electrohydraulic crusher and the stone pieces were taken out (22).

After penetrating the urethra scope into patient urinary tract and crushing process, 5-10 cc of urine was collected from each kidney. A 5 minutes centrifugation at $3000 \mathrm{rpm}$ was performed to separate the blood resulting from the crushing process.

To measure the levels of calcium, uric acid, and creatinine, an AutoAnalyzer (Mindray Company, BS-360 Model) and laboratory kits (Bionic Company) were used. The samples were directly tested to measure uric acid and calcium. In order to measure oxalate and citrate levels, a manual protocol was run using manual kits (Darman Faraz Kave).

To measure oxalate levels according to the protocols 
of these kits, an enzyme that converts urinary oxalate to carbon dioxide and hydrogen peroxide is used. Then, the produced hydrogen peroxide makes a colored complex in the presence of peroxidase by oxidizing the proper chromogen, and is spectrophotometrically measured. The color intensity represents oxalate concentration.

Citrate lyase produces alpha-galactosidase that immediately reacts with phenyl hydrazine in acidic $\mathrm{pH}$ and produces phenyl hydrazine. The resulting compound has an absorbance of $340 \mathrm{~nm}$ wavelength that is proportionate to the citrate concentration.

\section{Ethical issues}

The research followed the principles of the Declaration of Helsinki. All patients provided informed consent to participate in the study. The protocol of this study was approved by the Ethics Committee of Shahrekord University of Medical Sciences (code: IR.SKUMS. REC.1394.45). The purposes of the study were explained to the participants and they were assured that their information would be kept confidential. This study was derived from a research project approved at the Deputy of Research and Technology of Shahrekord Medical University (grant \# 224.8).

\section{Data analysis}

The results on both healthy and stone-generating kidneys of each patient were separately recorded. Statistical analysis was performed by descriptive (central tendency indices and distribution of data) and inferential (z-test, chi-square, and Fisher's exact tests) statistics.

It is generally argued that metabolic defects are less likely to occur in the first time stone formers than in the patients with recurrent disease. Therefore, we comprehensively evaluated the urinary abnormalities in patients with kidney stone. It should be mentioned that $P$ value $<0.05$ was considered the significance level.

\section{Results}

In this study, 40 patients with unilateral urolithiasis were investigated of whom 29 were male (\%75.5). The mean age of our participants was $42.9 \pm 10.74$ (range: $18-74$ ) years. The mean duration of suffering from kidney stone was $4.46 \pm 2.31$ (range: $1-10$ ) years in our participants. Around $42.5 \%$ of the patients had first degree relatives with the history of kidney stone. Thirty individuals (75\%) lived in cities and 10 individuals (25\%) lived in rural areas.
For living place, 18 (45\%) patients lived in Shahrekord county, 9 (22.5\%) in Farsan, 4 (10\%) in Ben, 3 (7.5\%) in Ardal, 3 (7.5\%) in Boroujen, and 1 (2.5\%) in Saman, Lordegan, and Koohrang.

In the current study, the levels of calcium, uric acid, and oxalate and also citrate/creatinine ratio were calculated for both healthy and stone-generating kidneys (Table 1). As Table 1 demonstrates, the difference in the mean ratio of uric acid, oxalate, and citrate to creatinine in the healthy kidneys and stone-generating kidneys was not significant. However, the mean UCa/UCr ratio in the healthy kidneys was $0.27 \pm 0.07$ and relatively greater than that in the stonegenerating kidneys $(0.11 \pm 0.04)$.

\section{Discussion}

In the present study, the urine biochemical characteristics were studied to investigate the urinary calculi because the investigation of the etiology of renal stones is based on urine chemistry rather than analysis of the stone itself. In fact, the accuracy of stone analysis is generally poor and, even when it is accurate, does not explain the pathophysiology of stone formation (4).

A difference was seen in the mean $\mathrm{UCa} / \mathrm{UCr}$ ratio between the healthy kidneys and the stone-generating kidneys with a marginal difference $(P=0.06)$. However, this difference can be valuable clinically, and increase in significance level of this ratio is likely with increasing the sample size.

In $67.5 \%$ (n: 27 ) of the patients, the $\mathrm{UCa} / \mathrm{UCr}$ ratio of the healthy kidneys was greater than that of the stonegenerating kidneys, which can highlight the value of this difference from clinical perspective. To the best of our knowledge, the study of Lee et al was the only study performed using the same method to our procedure, which showed the amount of the released calcium from the healthy kidneys within 24 hours is more than that from the stone-generating kidneys in the urine of 13 individual with unilateral urolithiasis. Since the urinary calcium level during 24 hours has a direct association with random $\mathrm{UCa} / \mathrm{UCr}$ ratio, this finding is in line with the results of the present study and inconsistent with those speculations that consider the release of large calcium amounts and calcium crystal formation as the reason for calcium kidney stone formation.

In the study of Lee et al, it was suggested that the obstruction due to kidney stone formation caused tubular damage in the obstructed kidney that reduced the calcium

Table 1. Comparison of the biochemical parameters of stone-generating kidneys and healthy kidneys in patients with unilateral urolithiasis

\begin{tabular}{lcc}
\hline Biochemical parameters & $\begin{array}{c}\text { Healthy kidney } \\
\text { (Mean } \pm \text { SD) }\end{array}$ & $\begin{array}{c}\text { Stone-generating kidney } \\
\text { (Mean } \pm \text { SD) }\end{array}$ \\
\hline Creatinine/calcium ratio & $0.27 \pm 0.07$ & $0.11 \pm 0.04$ \\
Uric acid/creatinine ratio & $0.44 \pm 0.07$ & $0.53 \pm 0.07$ \\
Oxalate/creatinine ratio & $0.06 \pm 0.03$ & $0.06 \pm 0.03$ \\
Citrate/creatinine ratio & $0.013 \pm 0.017$ & $0.015 \pm 0.019$ \\
\hline
\end{tabular}


release from the obstructed kidney tubules. Besides that, it was already mentioned that in animal models, in case of kidney obstruction, the healthy kidney releases more calcium as a compensatory mechanism that can be a risk factor for the formation of kidney stone in the healthy kidney in the following years.

Since the materials and types of stones were not determined in our patients, in certain cases, it is likely that the cause of kidney stone is not associated with metabolic disorders in the calcium release in the kidneys. We observed certain stones such as the uric acid stones, which can be the reason for the increase in $\mathrm{UCa} / \mathrm{UCr}$ ratio in the healthy kidney.

Better et al reported that for patients with complete unilateral obstruction, the clearance of creatinine reached from 2.6 to $10.2 \mathrm{~mL} / \mathrm{min}$ during a week after obstruction removal, and remained unchanged for four weeks (24). The study of Jones et al also demonstrated that the function of the kidney with unilateral obstruction returned to the condition before obstruction through two phases following obstruction removal (25).

It is therefore obvious that for the close investigation of the released calcium from the stone-generating kidneys, at least two weeks are needed for the kidney to function normally again. Since our study was conducted on humans, there was no possibility, from ethical and practical perspectives, to investigate stone-generating kidney function after the obstruction removal. Because surgical intervention was needed to collect separate specimens from the patient kidneys again. It is expected that after removing obstruction, the calcium release from the stone-generating kidney increases over time. Even, it is likely that larger amounts of calcium are released in the stone-generating kidney specimens.

In a study, the level of $\mathrm{UCa} / \mathrm{UCr}$ ratio was higher in the urinary stone formers group than the control group. They concluded that it can be a useful and comparatively easier method in the first level examination of urinary stone formers (13).

The mean $\mathrm{UCa} / \mathrm{UCr}$ ratio of non-fasting random urinary samples was obtained $0.123 \pm 0.106$ in a study in south Iran with healthy children (26).

A study on healthy children in north Iran showed the mean UCa/UCr ratio in girls to be $0.16 \pm 0.1$ and in boys to be $0.15 \pm 0.09$, with an inverse correlation between this ratio and age (27). The reason for different values reported for this variable can be due to the differences in geographical regions.

In the study of Ceran et al, the mean UCa/UCr ratio had an inverse correlation with age, while the geographical location of the subjects was found to be effective on this ratio (28).

In our study, no significant difference in mean of uric acid/creatinine ratio in the healthy kidneys and stonegenerating kidneys was detected. In 70\% (n: 28) of patients, the acid uric/creatinine ratio in the healthy kidneys was greater than that in the stone-generating kidneys; and in $30 \%$ (n: 12 ), this ratio was higher in the stone-generating kidneys. However, the mean uric acid/creatinine ratio was greater in stone-generating kidneys, which can confirm the previous findings regarding the uncertainty of stone material. It seems that a number of patients had uric acid stones.

In the study of Jawalekar et al, no significant difference was seen in the mean uric acid/creatinine ratio between the patients with kidney stone and healthy people. However, in this research, 24 hours urine uric acid level of patients with kidney stone was greater than that of the control group (18).

From another perspective, uric acid can create the primary core of calcemic stones in heterogeneous crystallization (29). Therefore, since the mean uric acid/ creatinine ratio was higher in the stone-generating kidneys, it can lead to formation of calcium stones in the stone-generating kidneys of the patients in our study.

In this study, no significant difference was seen in the mean oxalate/creatinine ratio and citrate/creatinine ratio between the people with healthy kidneys and those with kidney stone. The significant level and minimal difference in these values between the two groups demonstrate that the secretion of citrate and oxalate is not a factor for unilateral urolithiasis. There has yet been no research on the investigation of difference in oxalate excretion and citrate excretion between the stone-generating kidney and healthy kidney in patients with unilateral urolithiasis. In previous studies, these variables have been compared between healthy people and patients. In addition, increased urinary calcium excretion significantly decreased urinary oxalate excretion $(P=0.05)$. The greatest crystal mass is produced when calcium and oxalate are present in urine at a 1:1 ratio (30). It was demonstrated that oxalate plays the most important role as urinary stone risk factor followed by calcium and uric acid. In addition to the risk factors, it seems that supersaturation as the sum of all risk factors probably has a high predictive value (31).

In the previous research, oxalate/creatinine ratio was presented as an indicator of hyperoxaluria, and the oxalate/ creatinine ratio was significantly greater in patients than in the healthy people (18). It can be argued that oxalate secretion cannot be affected by external factors while, those factors exclusively related to kidney stone, and its excretion was mainly affected by metabolic systemic factors. No significant difference was seen between the oxalate excretion in the healthy kidney and that in the stone-generating kidney. Sikora et al, considered citrate to be the most important protective factor against kidney stone formation (32).

Another study indicated that the kidney appears to be involved in the pathogenesis of hypocitraturia in kidney stone patients and that a substantial proportion of these patients show a reduced urinary excretion of citrate (18).

However, in the present study, with regard to the mean 
citrate/creatinine ratio in the healthy kidneys and the stone-generating kidneys $(0.015 \pm 0.0119$ and $0.013 \pm 0.017$, respectively) with a significant difference (0.93), citrate cannot be considered an effective factor for unilateral kidney stone formation.

The reason for the inconsistency in the results of this study and other studies might be comparison of the individuals with kidney stone with healthy individuals in the previous studies. However, in this study, the healthy kidney of a patient with unilateral urolithiasis was compared with his/her stone-generating kidney.

Several theories and factors were suggested for unilateral urolithiasis such as anatomic disorders or structural deformities in one kidney of the individuals. In this condition, the main role is related to the urinary stasis in a special point or disrupted urodynamic flow that makes the environment suitable for the formation of the primary crystals (21).

The other reason is the repetition or habits of the patients in sleeping position toward a specific side that predispose them to stone and crystal formation due to the blood flow that is under the influence of weight and gravity toward the kidney of the same side and increased renal perfusion (19).

Although the impact of sleeping position in recurrent unilateral stone formation remains unknown, sleep posture may play a role in promotion of urolithiasis (33).

\section{Conclusion}

The mean UCa/UCr ratio of the patients' healthy kidneys was greater than that of their stone-generating kidneys. However, no statistically significant difference was seen in the mean uric acid, oxalate, and citrate ratio between the healthy kidneys and the stone-generating kidneys. A significant difference was seen in the mentioned variables between the individuals with kidney stone and healthy individuals in the previous studies.

However, in the present research, the healthy kidney and the stone-generating kidney of a patient were investigated, which demonstrated that unilateral urolithiasis did not have any direct association with topical factors and the secretory disorders of the stone-generating kidney in comparison with healthy kidney. Therefore, other effective factors such as metabolic factors, frequent positions during 24 hours and sleep, and anatomic disorders of the stone-generating kidney should be studied. These factors, in addition to topical secretory disorders, cause the development and even recurrence of unilateral urolithiasis.

With regards to the lack of similar research to investigate the healthy and unhealthy kidneys in patients with unilateral urolithiasis, further studies with larger sample size with respect to demographic variables are recommended.
Limitations of the study

The small sample size was one of the limitations of the study. It is recommended to conduct the study with the more samples.

\section{Authors' distribution}

MS; Study concept and design, acquisition of data, analysis and interpretation of data; FM; Study concept, drafting of the manuscript; HS; critical revision of the manuscript for important intellectual content, administrative, technical, and material support.

\section{Conflicts of interest}

The authors declared no competing interests.

\section{Ethical considerations}

Ethical issues (including plagiarism, data fabrication, double publication) have been completely observed by the authors.

\section{Funding/Support}

This paper was derived from a research project approved at the Deputy of Research and Technology of Shahrekord Medical University (grant \# 224.8); hereby, the financial and moral support of this university is appreciated.

\section{References}

1. Tiselius HG, Daudon M, Thomas K, Seitz C. Metabolic work-up of patients with urolithiasis: indications and diagnostic algorithm. Eur Urol Focus. 2017;3:62-71. doi: 10.1016/j.euf.2017.03.014.

2. Basiri A, Shakhssalim N, Khoshdel AR, Naghavi M. Regional and seasonal variation in the incidence of urolithiasis in Iran: a place for obsession in case finding and statistical approach. Urol Res. 2009;37:197-204. doi: 10.1007/s00240-009-0193-5

3. Dursun I, Poyrazoglu HM, Dusunsel R, Gunduz Z, Gurgoze MK, Demirci D, et al. Pediatric urolithiasis: an 8-year experience of single centre. Int Urol Nephrol. 2008;40:3-9. PubMed PMID: 17611811. doi: 10.1007/s11255-007-92346

4. Jones AN, Shafer MM, Keuler NS, Crone EM, Hansen KE. Fasting and postprandial spot urine calcium-to-creatinine ratios do not detect hypercalciuria. Osteoporos Int. 2012;23:553-62. doi: 10.1007/s00198-011-1580-7.

5. Safarinejad MR. Adult urolithiasis in a population-based study in Iran: prevalence, incidence, and associated risk factors. Urol Res. 2007;35:73-82. doi: 10.1007/s00240-0070084-6

6. Sakhaee K, Maalouf NM, Sinnott B. Clinical review. Kidney stones 2012: pathogenesis, diagnosis, and management. J Clin Endocrinol Metab. 2012;97:1847-60. doi: 10.1210/ jc.2011-3492.

7. Hadjzadeh MA, Khoei A, Hadjzadeh Z, Parizady M. Ethanolic extract of nigella sativa $\mathrm{L}$ seeds on ethylene glycol-induced kidney calculi in rats. Urol J. 2007;4:86-90.

8. 8.Vijaya T, Kumar MS, Ramarao NV, Babu AN, Ramarao N. Urolithiasis and its causes-short review. J Phytopharmacol. 
2013;2:1-6

9. Saitz TR, Mongoue-Tchokote S, Sharadin C, Giel D, Corbett S, Kovacevic L, et al. 24 hour urine metabolic differences between solitary and recurrent stone formers: results of the Collaboration on Urolithiasis in Pediatrics (CUP) working group. J Pediatr Urol. 2017;13:506.e1-506.e5. doi: 10.1016/j. jpurol.2017.03.015.

10. Pak CY, Poindexter JR, Adams-Huet B, Pearle MS. Predictive value of kidney stone composition in the detection of metabolic abnormalities. Am J Med. 2003;115:26-32.

11. Stoller ML. Urinary stone disease. In: Tanagho EA, McAninch JW, editors. Smith's General Urology. $16^{\text {th }}$ ed. New York: McGraw-Hill; 2004. pp. 256-86.

12. So NP, Osorio AV, Simon SD, Alon US. Normal urinary calcium/creatinine ratios in African-American and Caucasian children. PediatrNephrol. 2001;16:133-9.

13. Abdallah AEH, Abusharib AB, Elbashir AM, Nourien IH. Utility of urinary calcium/ creatinine ratio in evaluation of urinary stone formers: a Sudanese case control study. Int J Res Med Sci. 2017;5:146-9.

14. Bultitude M, Rees J. Management of renal colic. BMJ. 2012;345:e5499. doi: 10.1136/bmj.e5499.

15. Furrow E, Patterson EE, Armstrong PJ, Osborne CA, Lulich JP. Fasting urinary calcium-to-creatinine and oxalate-tocreatinine ratios in dogs with calcium oxalate urolithiasis and breed-matched controls. J Vet Intern Med. 2015;29:1139. doi: 10.1111 /jvim.12527.

16. Tiselius HG. A hypothesis of calcium stone formation: an interpretation of stone research during the past decades. Urol Res. 2011;39:231-43. doi: 10.1007/s00240-010-0349-3.

17. Green DF, Lytton B. Early experience with direct vision electrohydraulic lithotripsy of ureteral calculi. J Urol. 1985;133:767-70.

18. Jawalekar SL, Kulkarni UJ, Surve VT, Bhutey A. Evaluation of different urinary constituent ratios in renal stone formers. Ann Biol Res. 2010;1: 50-5.

19. Shekarriz B, Lu HF, Stoller ML. Correlation of unilateral urolithiasis with sleep posture. J Urol. 2001;165:1085-7.

20. Xie B, Halter TJ, Borah BM, Nancollas GH. Aggregation of Calcium Phosphate and Oxalate Phases in the Formation of Renal Stones. Cryst Growth Des. 2015;15(:204-11. doi:10.1021/cg501209h.

21. Gambaro G, Fabris A, Puliatta D, Lupo A. Lithiasis in cystic kidney disease and malformations of the urinary tract. Urol
Res. 2006;34:102-7. doi: 10.1007/s00240-005-0019-Z

22. Falahatkar S, Khosropanah I, Allahkhah A, Jafari A. Open surgery, laparoscopic surgery, or transureteral lithotripsy-which method? Comparison of ureteral stone management outcomes. J Endourol. 2011;25:31-4. doi: 10.1089/ end.2010.0344.

23. Lee YH, Chang SS, Chen MT, Huang JK, Huang WC.Reduction of calcium excretion in the stone-forming kidney in unilateral ureteral obstruction. Urol Res. 1991;19:193-8.

24. Better OS, Arieff AI, Massry SG, Kleeman CR, Maxwell $\mathrm{MH}$. Studies on renal function after relief of complete unilateral ureteral obstruction of three months' duration in man. Am J Med. 1973;54:234-40.

25. Jones DA, George NJ, O'Reilly PH, Barnard RJ. The biphasic nature of renal functional recovery following relief of chronic obstructive uropathy. Br J Urol. 1988;61:192-7.

26. Emamghorashi F, Davami MH, Rohi R. Hypercalciuria in Jahrom's school-age children: what is normal calciumcreatinine ratio? Iran J Kidney Dis. 2010;4:112-5.

27. Sorkhi H, Haji Aahmadi M. Urinary calcium to creatinine ratio in children. Indian J Pediatr. 2005 Dec;72:1055-6.

28. 28.Ceran O, Akin M, Akturk Z, Ozkozaci T. Normal urinary calcium/creatinine ratios in Turkish children. Indian Pediatr. 2003;40:884-7.

29. 29.Berg C, Tiselius HG. The effects of citrate on hydroxyapatite induced calcium oxalate crystallization and on the formation of calcium phosphate crystals. Urol Res. 1989;17:167-72.

30. 30. Marangella M, Vitale C, Petrarulo M, Bagnis C, Bruno M, Ramello A. Renal stones: from metabolic to physicochemical abnormalities. How useful are inhibitors? J Nephrol. 2000;13 Suppl 3:S51-60.

31. 31.Parvin M, Shakhssalim N, Basiri A, Miladipour AH, Golestan B, MohammadiTorbati P, et al.The most important metabolic risk factors in recurrent urinary stone formers. Urol J. 2011;8:99-106.

32. 32. Sikora P, Bienias B, Majewski M, Borzecka H, Wawrzyszuk M, Zajaczkowska M. [Urinary citrate excretion in children with calcium urolithiasis]. PrzeglLek. 2006;63 Suppl 3:134-6.

33. 33. Ziaee SA, Moradi A, Fateh M, Moghaddam SM. Sleep posture and unilateral renal stone formation. Scand J Urol Nephrol. 2008;42:551-4.

Copyright $\odot 2019$ The Author(s); Published by Nickan Research Institute. This is an open-access article distributed under the terms of the Creative Commons Attribution License (http://creativecommons.org/licenses/by/4.0), which permits unrestricted use, distribution, and reproduction in any medium, provided the original work is properly cited. 